\title{
Transition from regular to complex behaviour in a discrete deterministic asymmetric neural network model
}

\author{
A Crisanti $\dagger$, M Falcioni $\dagger \ddagger$ and A Vulpiani $\dagger \ddagger$ \\ † Dipartimento di Fisica, Università di Roma "La Sapienza", I-00185 Roma, Italy \\ $\ddagger$ INFN Sezione di Roma \\ PACS number(s): 05.45.+b, 87.10.+e
}


Abstract. We study the long time behaviour of the transient before the collapse on the periodic attractors of a discrete deterministic asymmetric neural networks model. The system has a finite number of possible states so it is not possible to use the term chaos in the usual sense of sensitive dependence on the initial condition. Nevertheless, at varying the asymmetry parameter, $k$, one observes a transition from ordered motion (i.e. short transients and short periods on the attractors) to a "complex" temporal behaviour. This transition takes place for the same value $k_{\mathrm{c}}$ at which one has a change for the mean transient length from a power law in the size of the system $(N)$ to an exponential law in $N$. The "complex" behaviour during the transient shows strong analogies with the chaotic behaviour: decay of temporal correlations, positive Shannon entropy, non-constant Renyi entropies of different orders. Moreover the transition is very similar to that one for the intermittent transition in chaotic systems: scaling law for the Shannon entropy and strong fluctuations of the "effective Shannon entropy" along the transient, for $k>k_{\mathrm{c}}$. 


\section{Introduction}

The properties of the low temperature phase of spin systems with random quenched couplings have been object of intesive analysis in the last years [Mézard et al.1987, Fischer and Hertz 1991]. The interest for such models comes from the study of both spin glasses and neural networks [Amit 1989]. One of the best known among these models, the Serrington - Kirkpatrick (SK) model, is a fully connected version of a spin glass. Here the interactions between pairs of spins are random, quenched and symmetric. This leads to a large number of metastable states which are responsible for the complicated dynamical behaviour of this and related models.

From a biological point of view the constraint of symmetric interactions is unsatisfactory. In fact, in the language of neural networks, two neurons will not act on each other in a symmetric way. It is then natural to investigate models with asymmetric couplings between spins [Amit 1989].

Apart from these biological motivations, the study of disordered spin systems with asymmetric bonds is also an interesting problem from the viewpoint of non equilibrium statistical mechanics. In fact for these models one no longer has a Hamiltonian and a detailed balance condition, and therefore no fluctuation dissipation theorem exists [Crisanti and Sompolinsky, 1987]. This last relation between the response and the correlation functions is, however, essential for extracting the long time properties of the dynamics. As a consequence, the long time limit has to be calculated via the full dynamic problem and cannot be evaluated by equilibrium statistical mechanical averages.

Till now such models have been studied principally in the framework of neural networks model, and the interest has been mainly focused on the properties of the attractors of networks of spins with asymmetric couplings. In particular the effect of the degree of asymmetry on the long time behaviour has been analysed. Although analytical results, based on perturbation expansions in the nonlinearity parameter of soft spin models [Crisanti and Sompolinsky 1987], showed a drastic change in the deterministic dynamics as soon as the asymmetry was switched on, subsequent numerical data are in agreement with a transition at a finite degree of asymmetry [Spitzner and Kinzel 1989]. A perturbation expansion in the asymmetry parameter for the hard Ising spin seems to suggest a transition at a finite value of the asymmetry 
parameter [Rieger et al.1989]. The location of the transition is, however, controversial.

An interesting feature of these models is the presence of extremely long transients [Crisanti and Sompolinsky 1988, Nützel 1991], i.e. the time the system wanders around before it settles down in the "asymptotic" regime. It has been found that while for nearly-symmetric networks the typical number of updates, before the relaxation onto the attractor, grows as a power of the system size, for strong enough asymmetry the transients are typically exponential in the size of the system [Nützel 1991]. Therefore it is natural to study the properties of the transient, because they give the features of a large enough system during any reasonable time.

In this paper we investigate the deterministic parallel dynamics of a fully connected Ising spin system with random quenched asymmetric interactions. We stress that if the spins are continuous, then this system exhibits chaotic behaviour, e.g. positive Lyapunov exponent, in the limit of high asymmetry [Sompolinsky et al.1988, Tirozzi and Tsodyks 1991]. In the case of Ising spins, however, because of the discrete nature of the states, it is not possible to characterize the time behaviour of the system by a straigthforword application of the most simple methods of chaotic dynamics, e.g. by the computation of the Lyapunov exponents. Nevertheless one can perform a study

of the temporal evolution in terms of well defined quantities, such as Shannon and Renyi entropies, which give a non ambiguous degree of the "complexity" of the system. From these indicators and from the behaviour of the correlation function one has a strong evidence for a transition, at varying the asymmetry parameter, from regular to "complex" behaviour in systems of finite size. This transition is very similar to the onset of turbulence in the intermittent scenario in chaotic dynamical systems [Pomeau and Manneville 1980].

The model will be described in Sect. 2; Sect. 3 deals with the chaotic - i.e. complex, in the information theory meaning — behaviour during the transient and in Sect. 4 we study the fluctuations of the chaotic degree along the trajectories, in terms of the Renyi entropies. In Sect. 5 the reader will find a summary and conclusions.

\section{The model}

In this paper we consider an asymmetric spin glass model with deterministic dynamics [Crisanti and Sompolinsky 1987]. The model consists of $N$ fully connected Ising spin 
$\sigma= \pm 1$ interacting via quenched random asymmetric couplings of the form

$$
J_{i j}=J_{i j}^{\mathrm{S}}+k J_{i j}^{\mathrm{A}}, \quad k \geq 0
$$

where $J_{j i}^{\mathrm{S}}=J_{i j}^{\mathrm{S}}$ and $J_{j i}^{\mathrm{A}}=-J_{i j}^{\mathrm{A}}$, and $(i, j)$ denotes a pair of spins. The diagonal elements $J_{i i}^{\mathrm{S}}$ are zero. The elements of the upper triangular part of the symmetric and antisymmetric matrices $\mathbf{J}^{\mathrm{S}}$ and $\mathbf{J}^{\mathrm{A}}$ are random independent Gaussian variables with zero mean and mean square equal to $1 /(N-1)\left(1+k^{2}\right)$ so that the rows of the couplings matrices have average norm 1 independently of the system size or symmetry.

The parameter $k$ defines the symmetry of the coupling, and ranges from $k=0$ for symmetric through $k=1$ for asymmetric to $k=\infty$ for antisymmetric couplings. In the literature it is often used the related parameter $\lambda$ defined by

$$
\lambda=\frac{1-k^{2}}{1+k^{2}}=N\left[J_{i j} J_{j i}\right]
$$

ranging from $\lambda=1$ for symmetric through $\lambda=0$ for asymmetric to $\lambda=-1$ for antisymmetric matrices. In (2.2) square brakets denote average over the realization of the coupling matrix $\mathbf{J}$.

Our analysis is restricted to deterministic parallel dynamics, i.e. the spins are updated simultaneously according to

$$
\sigma_{i}(t+1)=\operatorname{sign}\left[\sum_{j=1}^{N} J_{i j} \sigma_{j}(t)\right] \text {. }
$$

For any given sample $\mathbf{J}$ the dynamics is deterministic and depends only on $\mathbf{S}(0)$ the spin configuration $\left\{\sigma_{i}\right\}$ at time $t=0$.

For a system of $N$ spins there are $2^{N}$ different possible states. The evolution $(2.3)$ connects these states among them. Therefore for each realization of the couplings the structrure of the dynamics can be depicted in terms of oriented graphs, i.e. a set of points representing the states connected by arrows indicating the transitions. An example is shown in Fig. 1. Obviously each state has one and only one outgoing arrow, but different arrows can end at the same state. Since the number of states is finite and the dynamics is deterministic, each initial configuration of spins evolves to a definite attractor, which can be either a fixed point $\mathbf{S}(t+1)=\mathbf{S}(t)$, or a periodic repetition of $l$ configurations $\mathbf{S}(t+l)=\mathbf{S}(t)$ (cycle of length $l$ ). 
From this remark one may be tempted to conclude that the behaviour of (2.3) is rather "trivial" in the sense that there is no possibility of having something similar to a chaotic behaviour. This is not completely true. In fact (2.3) can be seen as a cellular automaton, for which the behaviour just described is common. Nevertheless, it is well known that there is a wide class of cellular automata with rather "complex" behaviours.

The reason is that the time it takes to reach the attractor can be very long. As a consequence the system may exhibit for a long time a rather complex behavior before it eventually relaxes to the "asymptotic" regime, usually a cycle. The presence of long transients, exponential in the system size, was first observed for sequential dynamics [Crisanti and Sompolinsky 1988] and recently analysed in more details for both sequential and parallel dynamics [Nützel 1991].

From the above considerations it follows that in these models there are two relevant time scales: the relaxation time $\tau$ and the cycle length $l$. These are defined as

$$
\begin{gathered}
l=\min _{n}[\mathbf{S}(t+n)=\mathbf{S}(t)] \\
\tau=\min _{n}[\mathbf{S}(l+n)=\mathbf{S}(n)] .
\end{gathered}
$$

The time $\tau$ is the time the system needs to reach the first cycle of length $l$. In general both $l$ and $\tau$ depend on the initial state $\mathbf{S}(0)$ and on the coupling realization $J_{i j}$. For any given $k$ and system size $\mathrm{N}$ one can define the average transient time and the average cycle length. To this end one considers a large number $M$ of independent coupling realizations $J_{i j}$. For each sample and a random initial state $\mathbf{S}(0)$ the time $\tau$ and the cycle length $l$ are recorded. One then defines the average values as

$$
\begin{aligned}
& \langle l\rangle=\frac{1}{M} \sum_{m=1}^{M} l(m) \\
& \langle\tau\rangle=\frac{1}{M} \sum_{m=1}^{M} \tau(m)
\end{aligned}
$$

where $l(m)$ and $\tau(m)$ are the values of $l$ and $\tau$ defined in (2.4) and (2.5) for the $m$-th sample.

Studying these average quantities as a function of both the symmetry and the system size, Nützel found strong numerical evidence for a transition between two 
different behaviours as the symmetry of the couplings is varied. He found that for high symmetric couplings $\langle l\rangle$ is size independent and $\langle\tau\rangle$ grows as a power of $N$, while the behaviour of both quantities is exponential in $N$ for high asymmetric couplings. He located the transition point around $\lambda \simeq 0.5$ where $\langle l\rangle$ shows strong changes [Nützel 1991]. By contrast $\langle\tau\rangle$ changes smoothly with $\lambda$. This behaviour was found for both sequential and parallel dynamics.

The values of $l$ and $\tau$, however, exhibit strong sample to sample fluctuations. As a consequence the leading contributions to the average (2.6) and (2.7) come from rare events. This suggests that the appropriate quantity for averaging is not $\tau$ and $l$, but their logarithms. One then defines the "typical" values

$$
\begin{aligned}
& \tau_{\text {typ }}=\exp \langle\ln \tau\rangle \\
& l_{\text {typ }}=\exp \langle\ln l\rangle .
\end{aligned}
$$

An analysis of these quantities as a function of both the symmetry of the couplings and the system size reveals that

$$
\tau_{\text {typ }} \sim\langle\tau\rangle \propto N^{\alpha(k)} \mathrm{e}^{\beta(k) N}
$$

where $\beta(k) \ll 1$ for $k_{\mathrm{c}} \approx 0.5$. A similar behaviour is observed for the cycle length $l$.

We find that, for $k \geq k_{\mathrm{c}}, \tau$ has a roughly lognormal distribution whose peak and width scale linearly with $N$. In other words $\ln \tau$ is a gaussian with

$$
\begin{gathered}
\langle\ln \tau\rangle \propto N \\
\left\langle(\ln \tau)^{2}\right\rangle-\langle\ln \tau\rangle^{2} \propto N^{2} .
\end{gathered}
$$

The same result remains true also for sequential dynamics [Crisanti and Sompolinsky 1988]. The lognormal distribution is quite common in disordered systems [Paladin and Vulpiani 1987]. However, in these models one has a non standard scaling of the variance of $\ln \tau$. In fact usually both the mean value and the variance scale with $N$. This means that here there are enormous fluctuations: the ratio between the standard deviation and the mean value is constant instead of being proportional to $N^{-1 / 2}$ as in the standard case.

The presence of these long transients, e.g. for $k=0.8$ and $N=100$ one has $\tau_{\text {typ }}=O\left(10^{6}\right)$, makes natural to study the dynamics during the transient. Not only 
because this is the behaviour of the system on reasonable times, but also because this is the behaviour which is revealed by any mean-field study of these systems.

To analyze the temporal evolution one of the first quantities one usually studies are the time correlations. For a given initial state $\mathbf{S}(0)$ and coupling realization $J_{i j}$ we compute the following quantity

$$
C(t)=\frac{1}{N} \sum_{i=1}^{N} \overline{\sigma_{i}\left(t+t^{\prime}\right) \sigma_{i}\left(t^{\prime}\right)}
$$

where the bar denotes time average. The correlation $C(t)$ is evaluated taking into account only the data for the transient, i.e. times less than $\tau$. We remark that $C(t)$ is not averaged over different realizations of $J_{i j}$. From the comparison of several realizations and different $N$, it seems that $C(t)$ is a selfaveraging quantity that depends very weakly on $N$. On the contrary $c_{i}(t)=\overline{\sigma_{i}\left(t+t^{\prime}\right) \sigma_{i}\left(t^{\prime}\right)}$ changes at varying $i$; and there exist two different possibilities: $c_{i}(t)$ either decreases monotonically with $t$, or it shows strong oscillations with a decreasing envelope.

For $k=1$ (full asymmetric case) one has $C(t)=0$ for $t \geq 1$. The behaviours of $C(t)$ for $k<1$ are reported in Fig. 2a. Unfortunatelly one does not have a simple formula to fit $C(t)$ as a function of $t$ at different $k$, even if, for each $k$, the data seem consistent with a stretched exponential decay:

$$
C(t) \sim \exp \left[-a t^{b}\right] \text { with } b<1
$$

Nevertheless one can define a characteristic time, $\tau_{\mathrm{c}}$, as the time at which the envelope of $C(t)$ reaches a threshold value, e.g. $1 /$ e. Figure $2 \mathrm{~b}$ shows $1 / \tau_{\mathrm{c}}$ vs $k$; one has evidence for a transition at $k \approx 0.5$. The above scenario does not change using different values for the threshold.

\section{Chaotic behaviour during the transient}

It is known [Isola et al.1985] that in some dynamical systems with many degrees of freedom the transition from regular to chaotic motion is also signaled by a change, from power to exponential in the number of degrees of freedom, of the Poincarè return time. 
This result and the analysis of the correlation functions discussed in the previous section suggest the possibility of a "chaotic behaviour" during the transients for enough asymmetric couplings. This is in spite of the fact that a stationary periodic orbit is always found to be the final attractor. The reason to study in more details this chaotic behaviour follows from the fact that if the asymmetry is strong enough the transient increases exponentially with the system size. Therefore any practical investigation of the time evolution is necessarily limited to the transient. Moreover one can argue that the attractor might not be relevant for the dynamics since even in the presence of stable solutions, generic initial conditions relax towards it only after exponentially long transients [Crutchfield and Kaneko 1988].

Due to the discrete nature of the spin and to the full connectivity of the system a quantification of "chaos" during the transient is not at all straightfowrard. In fact, for systems whose state changes continuously one can quantify the degree of chaos in terms of the Lyapunov exponents [Benettin et al.1980a, b]. They measure the exponential growth rate of the distance between two initially close trajectories.

One can try to follow the same idea of the Lyapunov exponents studing two trajectories whose initial condition $\mathbf{S}(0)$ and $\mathbf{S}^{\prime}(0)$ differ by a single spin. Looking at the growth of the Hamming distance between $\mathbf{S}(t)$ and $\mathbf{S}^{\prime}(t)$ one can then introduce something similar to the first Lyapunov exponent. This is the socalled 'damage spreading' method which revealed useful for discrete systems similar to (2.3) but with only local interactions among the spins [Kauffman 1969, Derrida and Stauffer 1986].

In this paper to characterize the chaotic behaviour we use a completely different approach. Following the basic idea of Shannon in the information theory [Khinchin 1957], we look at the "complexity" of the temporal history on the $i$-th spin during the transient [Grassberger 1986]. For each initial configuration and fixed realization of $J_{i j}$ the "history life" of a spin $\sigma_{i}$ before the system relaxes to the attractor can be written as a sequence

$$
\sigma_{i}(0), \sigma_{i}(1), \ldots, \sigma_{i}(m)
$$

where $m<\tau+l$. Since the transient increases exponentially with the system size, in principle the length of the sequence (3.1) can be done as large as we want by increasing $N$.

The complexity of the temporal behaviour of the spin can be caracterized by looking at the average amount of information contained in the sequence (3.1). To this 
end we consider a sequence $\omega_{n}$ of $n$ possible outcomes of the spin $\sigma_{i}$. Since it can assume only two values there are $2^{n}$ possible sequences. These can be regarded as strings of $n$ binary digits. In general, the sequences $\omega_{n}$ will appear in the history life of the spin $\sigma_{i}$ with different frequencies. Some of them may appear not at all or with very small probability. By computing the frequency with which each $\omega_{n}$ appears in the sequence $(3.1)$ we can define the probability $P\left(\omega_{n}\right)$ that the dynamics generates the sequence $\omega_{n}$. The measure of information contained in the knowledge of the $P\left(\omega_{n}\right)$ is given by the entropy $H(n)$ defined as

$$
H(n)=-\sum_{\omega_{n}} P\left(\omega_{n}\right) \ln P\left(\omega_{n}\right)
$$

where the sum is over all the sequences $\omega_{n}$. If the sequence (3.1) is stationary the quantity $H(n)$ depends only on $n$ and not on the initial state $\sigma_{i}(0)$. In this case, in the limit of large $n$ the average information $H(n) / n$ converges to the limit value $h$ which is called the Shannon entropy of the sequence (3.1) [Khinchin 1957]. The existence of this limit implies that for large $n$ the mean gain of information in considering sequences of length $n+1$ instead of $n$, i.e.

$$
h(n)=H(n+1)-H(n)
$$

approaces a constant limit given by the Shannon entropy $h$.

If the sequence (3.1) is a Markov chain, then $h(n)=h$ for each $n$. This can be generalized to Markov chains of order $p$, i.e. the probability of having an event at time $\mathrm{t}$ depends only on the events at times $t-p$ up to $t-1$, in which case $h(n)=h$ for $n \geq p$.

The relevance of $h$ follows from the first Shannon - McMillan theorem [Khinchin 1957] which states that for large $n$ the number of $n$-term sequences $\omega_{n}$ one can really observe in (3.1) is

$$
N_{\text {eff }}(n) \sim \mathrm{e}^{h n}
$$

This can also be stated as follows. For $n$ sufficiently large all the $n$-term sequences $\omega_{n}$ can be separated into two classes $\Omega_{1}(n)$ and $\Omega_{2}(n)$, such that for every sequence in the first class $P\left(\omega_{n}\right) \simeq \exp (-h n)$ and

$$
\sum_{\omega_{n} \in \Omega_{1}(n)} P\left(\omega_{n}\right) \rightarrow 1 \text { for } n \rightarrow \infty
$$


On the contrary, for the sequences in the second class

$$
\sum_{\omega_{n} \in \Omega_{2}(n)} P\left(\omega_{n}\right) \rightarrow 0 \quad \text { for } n \rightarrow \infty
$$

If we remember that the maximum number of $n$-term sequences is

$$
N_{\max }(n)=2^{n}=\mathrm{e}^{n \ln 2}
$$

we see that if $h<\ln 2$ for large $n$ the class $\Omega_{1}(n)$ contains only a negligible fraction of sequences. On the contrary, the overwhelming majority of such sequences fall into $\Omega_{2}(n)$.

If the probability of having $\sigma_{i}=1$ or $\sigma_{i}=-1$ in (3.1) is the same, as it is in our case, then $h<\ln 2$ signals the presence of a rule in the sequence. Thus the Shannon entropy gives a measure of the "complexity" of the sequence. In particular if $h=0$ then we are in presence of periodic motion, while if $h=\ln 2$ the sequence is generated with an head and tail trial. We stress that $\ln 2$, the largest value attainable by $h$, is reached only if the symbols in (3.1) are independent and equiprobable. In fact $h<\ln 2$ also if the symbols are independent but not equiprobable, e.g. generated according an head and tail trial done with an unbalanced coin [Khinchin 1957].

To analyze the motion of the system during the transient, we have computed the Shannon entropy of different spins for a fixed, randomly chosen, configuration of couplings $J_{i j}$. In all the cases we have taken $N$ large enough to ensure sufficiently long transients: $\tau \sim O\left(10^{5}\right)$ at least. For for $k \geq 0.7$ we considered $N=100, N=200$ and $N=300$, while for $k<0.7$ we used $N=200$ and $N=300$. For smaller value of $k$ the required system size becomes very large and, since the computational time increases as $N^{2}$, it is practically impossible to reach too small values of $k$. In all the cases we checked that the sequence (3.1) for each spin is stationary.

By computing $h(n)$ for different $n$ we found a rapid convergence towards the Shannon entropy $h$. In Fig. 3 it is shown a typical case from which we see that $n=4$ or $n=5$ are sufficient to have a good extimate of $h$.

In the limit case of $k=1$ the evaluated $h$ is very close to the largest value $\ln 2$. This is not surprising since if the couplings $J_{i j}$ and $J_{j i}$ are uncorrelated then the statistical properties of the dynamical system (2.3) are the same as the sequence generated by an head and tail trial [Gutfreund et al.1988]. 
For a given configuration of couplings $J_{i j}$ and a fixed value of $N$ we have computed the Shannon entropy $h^{(i)}$ for each sequence (3.1). The mean value

$$
\langle h\rangle=\frac{1}{N} \sum_{i=1}^{N} h^{(i)}
$$

seems to be a selfaveraging quantity, which does not depend on $N$; moreover the variance

$$
\frac{1}{N} \sum_{i=1}^{N}\left(h^{(i)}-\langle h\rangle\right)^{2}
$$

decreases for increasing $N$. In Fig. 4 we show $\langle h\rangle^{2}$ as a function of $k$ : it is well evident the scaling

$$
\langle h\rangle \sim\left(k-k_{\mathrm{c}}\right)^{\frac{1}{2}} \text { with } k_{\mathrm{c}}=0.50
$$

We close this section with a remark. Note that the point at $k=0.56$ does not agree very well with Eq. (3.5). Since it is practically impossible for us to work with smaller values of $k$, we cannot say if this point can be considered as an evidence for a crossover behaviour. In Sect. 5 we shall consider again this issue.

\section{Fluctuations during the transient}

In general not all the $2^{n}$ possible $n$-term sequences will appear in the sequence (3.1) generated by the dynamics. Nevertheless, for large $n$ their number $\widetilde{N}(n)$ increases exponentially with $n$. The rate of growth

$$
K_{0}=\lim _{n \rightarrow \infty} \frac{1}{n} \ln \tilde{N}(n)
$$

is the topological entropy. The smaller $K_{0}$ the larger the number of forbidden $n$-term sequences in (3.1). For our system we found that at any value of $k$ the topological entropy assumes its maximum value $K_{0}=\ln 2$. This means that for fixed $J_{i j}$ and generic initial condition, all possible sequences are actually generated by (2.3).

The topological entropy and the Shannon entropy are global quantities, since they ignore the finite time, i.e. finite $n$, fluctuations. For any finite $n$ we can define the "effective Sahnnon entropy" $\gamma(n)$ of the $n$-term string $\omega_{n}$ as

$$
\gamma(n)=-\frac{1}{n} \ln P\left(\omega_{n}\right) .
$$


In general the value of $\gamma(n)$ will depend on the string $\omega_{n}$, even for large but finite $n$. These finite $n$ fluctuations can be characterized by means of the Renyi entropy [Renyi 1970, Paladin and Vulpiani 1987]. Let us define

$$
H_{q}(n)=-\frac{1}{q-1} \ln \left[\sum_{\omega_{n}} P\left(\omega_{n}\right)^{q}\right]
$$

where the sum is extended over all the $2^{n} n$-term sequences $\omega_{n}$. In the limit of large $n$ the quantity

$$
h_{q}(n)=H_{q}(n+1)-H_{q}(n)
$$

or equivalently $H_{q}(n) / n$, converges towards the limit $h_{q}$ called Renyi entropy of order $q$.

From the definition it readly follows that Shannon and topological entropies are obtained from $h_{q}$ via $h=\lim _{q \rightarrow 1} h_{q}$ and $K_{0}=\lim _{q \rightarrow 0} h_{q}$. It is possible to show that in general $h_{q}$ is a not-increasing function of $q$ [Renyi 1970]. In the limit case of absence of fluctuations one has $h_{q}=K_{0}=$ constant. Thus the deviation of $h_{q}$ from $K_{0}$ gives a measure of the relevance of the fluctuations.

As we have done in Sect. 3 for the Shannon entropy, we have computed, for each sequence $\sigma_{i}(1), \sigma_{i}(2), \ldots$, the Renyi entropies $h_{q}^{(i)}$. For values of $k$ close to $1 h_{q}^{(i)}$ does not change very much at varying $q$, while for $k$ close to $k_{\mathrm{c}}$ one observes large deviations of $h_{q}^{(i)}$ from $h^{(i)}$. In Fig. 5 we show $\left\langle h_{q}\right\rangle$ as a function of $q$ for $k=0.9$ and $k=0.7$ : even at a qualitative level, the difference is well evident. The properties discussed in Sect. 3, for the Shannon entropy, hold true also for $h_{q}$ : each $\left\langle h_{q}\right\rangle$ is a selfaveraging quantity which does not depend on $N$.

The Renyi entropies are deeply related to the probability distribution $P_{n}(\gamma)$ of the "effective Shannon entropy" $\gamma(n)$. For large $n$ it is reasonable to make the ansatz that $P_{n}(\gamma)$ vanishes exponentially [Eckmann and Procaccia 1986, Paladin et al.1986],

$$
P_{n}(\gamma) \propto \exp [-n S(\gamma)], \quad S(\gamma) \geq 0
$$

The equal sign holds only for $\gamma=h$, as a consequence of the Shannon-McMillan theorem. With this assumption one finds

$$
h_{q}=\frac{1}{q-1} \min _{\gamma}[(q-1) \gamma+S(\gamma)] .
$$


This is a Legendre transform which shows that each $q$ selects a particular class of sequences with "effective Shannon entropy" $\gamma$. In particular $q=1$ selects the most probable sequences, whereas for $q \neq 1$ the leading contribution to $h_{q}$ comes from rare sequences.

For $\gamma \simeq h$ one has the following parabolic approximation for $S(\gamma)$

$$
S(\gamma)=\frac{(\gamma-h)^{2}}{2 \mu}
$$

where $\mu$ is given by

$$
\begin{aligned}
\mu & =\lim _{n \rightarrow \infty} \frac{1}{n}\left[\sum_{\omega_{n}} P\left(\omega_{n}\right)\left[\ln P\left(\omega_{n}\right)\right]^{2}-\left[\sum_{\omega_{n}} P\left(\omega_{n}\right) \ln P\left(\omega_{n}\right)\right]^{2}\right] \\
& =\lim _{n \rightarrow \infty} n \int(\gamma-h)^{2} P_{n}(\gamma) \mathrm{d} \mu .
\end{aligned}
$$

Equation (4.7) corresponds to

$$
h_{q}=h-\mu(q-1) / 2, \quad q \simeq 1
$$

one can consider $\mu$ as an inhomogeneity factor. The (4.7) and (4.8) correspond to a gaussian approximation. The quantities $h$ and $\mu$ are the most relevant caracterizations for the "complexity" of the sequence since $h$ gives the typical value and $\mu$ is related to the variance of the fluctuations.

We found that for $k$ close to $1 \mu$ assumes very small values, indicating that the sequences are almost homogeneous. When $k$ is decreased $\mu$ increases and assumes its largest values (strong fluctuations) as one reaches the critical value $k_{\mathrm{c}}$ In Fig. 6 we show $\langle\mu\rangle$ as a function of $k$.

It is interesting to note that the behavior of $\langle h\rangle$ and $\langle\mu\rangle$ as function of the parameter $k$ is similar to the one observed for the intermittent transition in dymical system, e.g. in the Lorenz model [Pomeau and Manneville 1980].

We close this section noting that all the results for $\langle h\rangle$ and $\langle\mu\rangle$ for $k$ larger than approximately 0.5 are practically independent of $N$ in the range $N=50 \div 500$. 


\section{Summary and conclusions}

In this paper we have studied the long-time behaviour of binary sequences, obtained from the deterministic evolution of an asymmetric neural networks model with $N$ components. The system size $N$ is large but finite. In spite of the discrete nature of its states, at varying the asymmetry parameter $k$, one observes some features very similar to the intermittent transition in chaotic systems. For $k<k_{\mathrm{c}}$ one has that the typical lenght of the transient follows a power law in the size of the system $N$, while for $k>k_{\mathrm{c}}$ the transients increase exponentially with $N$. One can define, in an unambiguous way, some degree of "complexity" during the transient, by using well established concept (Shannon and Renyi entropies) in the information theory.

For $k>k_{\mathrm{c}}$ the time behaviour of the transient is very similar to that one in the chaotic systems: decay of the temporal correlation and positive Shannon entropy. Around $k_{\mathrm{c}}$ one has a scaling law for the mean Shannon entropy $\langle h\rangle$ :

$$
\langle h\rangle \sim \sqrt{k-k_{\mathrm{c}}}
$$

moreover the fluctuations of the degree of chaos, given by the mean inhomogeneity factor $\langle\mu\rangle$, are large for $k$ approaching $k_{\mathrm{c}}^{+}$.

All our results on the Shannon and Renyi entropies and characteristic times for $k$ larger than approximately 0.5 do not depend on the value of $N$ in the investigated range $N=50 \div 500$. However, in principle, we cannot exclude the possibility that what we found is not a real sharp transition, but the manifestation, at finite $N$, of a crossover behaviour. For instance, for the sequential dynamics [Crisanti and Sompolinsky 1988] one has an exponential growth with $N$ for $\tau_{\text {typ }}$ according to (2.10) even for $k<k_{\mathrm{c}}$, but the constant $\beta(k)$ is so small that in order to see the exponential law one has to consider enormous systems, $N=O\left(10^{3}\right)$. In a symilar way in the above quoted reference the average correlation function during the transient has a characteristic

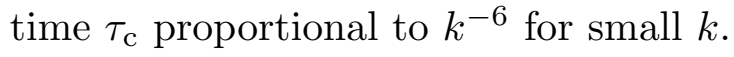

From our computations and the previous study of Nützel [1991] one can conclude that the dependence of $k_{\mathrm{c}}$ on $N$, if any, is very weak. For almost all the practical purposes, however, the nature of the transition, i.e. sharp or crossover, is not very important. Nevertheless, its existence is relevant for numerical studies. For example, it could be very dangerous to compute temporal averages at different $k$ using the same 
$N$ and maximal time. Indeed the meaning of these averages is completely different: for $k>k_{\mathrm{c}}$ one should have informations about the transient while for $k<k_{\mathrm{c}}$ one just observes properties of the periodic attractors.

We conclude by noting that similar problems are present also in hamiltonian (and more generally symplectic) systems with many degrees of freedom [Falcioni et al.1991].

\section{Acknowledgments}

We thank D J Amit, F Bagnioli, H Kunz and S Ruffo for useful discussions and suggestions. 


\section{References}

Amit D J 1989 Modeling brain function (Cambridge University Press)

Benettin G, Galgani L, Giorgilli A and Strelcyn J M 1980a Meccanica 159

-1980b Meccanica 1521

Crisanti A and Sompolinsky H 1987 Phys Rev A 364922

—1988 Phys Rev A 374865

Crutchfield J P and Kaneko K 1988 Phys Rev Lett 602715

Derrida B and Stauffer D 1986 Europhys Lett 2739

Eckmann J-P and Procaccia I 1986 Phys Rev A 34659

Falcioni M, Marini Bettolo Marconi U and Vulpiani A 1991 Phys Rev A 442263

Fischer K H and Hertz J A 1991 Spin Glasses (Cambridge University Press)

Grassberger P 1986 Int J Theor Phys 25907

Gutfreund H, Reger J D and Young A P 1988 J Phys A 212775

Isola S, Livi R and Ruffo S 1985 Phys Lett A 112448

Kauffman S A 1969 J. Theor. Biol. 22437

Khinchin A Ya 1957 Mathematical Foundations of Information Theory (Dover - New York)

Mézard M, Parisi G and Virasoro M A 1987 Spin Glass Theory and Beyond (World Scientific)

Nützel K 1991 J Phys. A 24 L151

Paladin G, Peliti L and Vulpiani A 1986 J Phys A 19 L991

Paladin G and Vulpiani A 1987 Phys Rep 156147

Pomeau Y and Manneville P 1980 Commun Math Phys 74189

Renyi A 1970 Probability Theory (North-Holland)

Rieger H, Schreckenberg M and Zittarz J 1989 Z Phys B 74527

Sompolinsky H, Crisanti A and Sommers H-J 1988 Phys Rev Lett 61259

Spitzner P and Kinzel W 1989 Z Phys B 77511

Tirozzi B and Tsodyks M 1991 Europhys Lett 14727 


\section{Figure captions}

Figure 1. Some examples of possible behaviour. One recognizes two fixed points and three cycles of period 2,3 and 4 , respectively.

Figure 2. a) $C(t)$ as a function of $t$ for $k=0.6$ (diamonds) and $k=0.8$ (plus); in order to evidenciate the main feature we plot $C(t)$ only at even values of $t$. b) $1 / \tau_{\mathrm{c}}$ as a function of $k$. The system size is $N=200$.

Figure 3. $h(n)$ as a function of $n$ for one spin sequence at $k=0.7$ and $N=200$.

Figure 4. $\langle h\rangle^{2}$ as a function of $k$ for $N=200$; the dashed line indicates the law $\langle h\rangle \propto\left(k-k_{\mathrm{c}}\right)^{1 / 2}$ with $k_{\mathrm{c}}=0.50$. The horizontal line corresponds to $\langle h\rangle=\ln 2$.

Figure 5. $\left\langle h_{q}\right\rangle$ as a function of $q$ for $k=0.9$ (plus) and $k=0.7$ (cross). The system size is $N=200$.

Figure 6. $\langle\mu\rangle$ as a function of $k$ for $N=200$. 